\title{
Time-dependent and bi-objective vehicle routing problem with time windows
}

\author{
Zhao, P.X. ${ }^{a,}{ }^{*}$, Luo, W.H. ${ }^{a}$, Han, X. ${ }^{a}$ \\ ${ }^{a}$ School of Management, Shandong University, Jinan, Shandong, P.R. China
}

\begin{abstract}
A B S T R A C T
The optimization of bi-objective vehicle routing problem has become a research hotspot in recent years. In this paper, a time-dependent and biobjective vehicle routing problem with time windows (TD-BO-VRPTW) is proposed, which is a new extension of classical vehicle routing problem. Time-dependency is presented for the situation that vehicle's travel speed is affected by its departure time and the distance between two customers. The total transportation costs and time costs are two objectives optimized simultaneously through constructing a bi-objective mixed integer linear programming model. To deal with this problem, the non-dominated sorting genetic algorithm II (NSGA-II) is adopted to obtain the Pareto optimal solution set. In the numerical examples, the RC108 from Solomon's benchmark set is employed and the results in the Pareto front show the efficiency of NSGA-II for the TD-BO-VRPTW. To further test the performance of this algorithm, two objectives are optimized separately and then the sum of two objectives is also optimized. Through comparing these results with solutions in the Pareto front, it can be concluded that the algorithm is reliable, and the results in Pareto front are competitive because there is a trade-off between two objectives.
\end{abstract}

\section{ARTICLE INFO}

Keywords:

Vehicle routing problem;

Time-dependency;

Bi-objective optimization;

Time windows;

Pareto optimal solutions;

Evolutionary algorithms;

NSGA-II algorithm

*Corresponding author:

pxzhao@sdu.edu.cn

(Zhao, P.X.)

Article history:

Received 14 February 2019

Revised 10 May 2019

Accepted 17 May 2019

\section{References}

[1] Dantzig, G.B., Ramser, J.H. (1959). The truck dispatching problem, Management Science, Vol. 6, No. 1, 80-91, doi: 10.1287/mnsc.6.1.80.

[2] Zhu, X.N., Yan, R., Zhang, Q. (2015). A promoted hybrid heuristic algorithm for two-dimensional multi-depots vehicle routing problem, International Journal of Simulation Modelling, Vol. 14, No. 3, 499-510, doi: 10.2507/ IISIMM14(3)CO11.

[3] Chiang, T.-C., Hsu, W.-H. (2014). A knowledge-based evolutionary algorithm for the multiobjective vehicle routing problem with time windows, Computers \& Operations Research, Vol. 45, 25-37, doi: 10.1016/i.cor.2013. 11.014.

[4] Solomon, M.M. (1987). Algorithm for the vehicle routing and scheduling problems with time window constraints, Operations Research, Vol. 35, No. 2, 254-265, doi: 10.1287/opre.35.2.254.

[5] Cao, Q.K., Yang, K.W., Ren, X.Y. (2017). Vehicle routing optimization with multiple fuzzy time windows based on improved wolf pack algorithm, Advances in Production Engineering \& Management, Vol. 12, No. 4, 401-411, doi: 10.14743/apem2017.4.267.

[6] Wu, D.Q., Dong, M., Li, H.Y., Li, F. (2016). Vehicle routing problem with time windows using multi-objective coevolutionary approach, International Journal of Simulation Modelling, Vol. 15, No. 4, 742-753, doi: 10.2507/IJSIMM15(4)C019.

[7] Yu, Y., Wang, S., Wang, J., Huang, M. (2019). A branch-and-price algorithm for the heterogeneous fleet green vehicle routing problem with time windows, Transportation Research Part B: Methodological, Vol. 122, 511-527, doi: 10.1016/i.trb.2019.03.009.

[8] Miranda, D.M., Conceição, S.V. (2016). The vehicle routing problem with hard time windows and stochastic travel and service time, Expert Systems with Applications, Vol. 64, 104-116, doi: 10.1016/i.eswa.2016.07.022. 
[9] Geiger, M.J. (2008). Genetic algorithms for multiple objective vehicle routing, arXiv:0809.0416, from https://arxiv.org/abs/0809.0416, accessed February 1, 2019.

[10] Barán, B., Schaerer, M. (2003). A multiobjective ant colony system for vehicle routing problem with time windows, In: Proceedings of the 21st IASTED International Conference on Applied Informatics, Innsbruck, Austria, 97102.

[11] Tan, K.C., Chew, Y.H., Lee, L.H. (2006). A hybrid multiobjective evolutionary algorithm for solving vehicle routing problem with time windows, Computational Optimization and Applications, Vol. 34, 115-151, doi: 10.1007/ s10589-005-3070-3.

[12] Ombuki, B., Ross, B.J., Hanshar, F. (2006). Multi-objective genetic algorithms for vehicle routing problem with time windows, Applied Intelligence, Vol. 24, No. 1, 17-30, doi: 10.1007/s10489-006-6926-z.

[13] Garcia-Najera, A., Bullinaria, J.A. (2011). An improved multi-objective evolutionary algorithm for the vehicle routing problem with time windows, Computers \& Operations Research, Vol. 38, No. 1, 287-300, doi: 10.1016/ j.cor.2010.05.004.

[14] Qi, Y., Hou, Z., Li, H., Huang, J., Li, X. (2015). A decomposition based memetic algorithm for multi-objective vehicle routing problem with time windows, Computers \& Operations Research, Vol. 62, 61-77, doi: 10.1016/ j.cor.2015.04.009.

[15] Iqbal, S., Kaykobad, M., Rahman, M.S. (2015). Solving the multi-objective vehicle routing problem with soft time windows with the help of bees, Swarm and Evolutionary Computation, Vol. 24, 50-64, doi: 10.1016/j.swevo. 2015.06.001.

[16] Xu, H., Fan, W., Wei, T., Yu, L. (2008). An Or-opt NSGA-II algorithm for multi-objective vehicle routing problem with time windows, In: Proceedings of 2008 IEEE International Conference on Automation Science and Engineering, Arlington, USA, 310-314, doi: 10.1109/coase.2008.4626505.

[17] Castro, J.P., Landa-Silva, D., Pérez, J.A.M. (2009). Exploring feasible and infeasible regions in the vehicle routing problem with time windows using a multi-objective particle swarm optimization approach, In: Krasnogor, N., Melián-Batista, M.B., Pérez, J.A.M., Moreno-Vega, J.M., Pelta, D.A. (eds.), Nature inspired cooperative strategies for optimization, (NICSO 2008), Studies in Computational Intelligence, Vol. 236, Springer, Berlin, Heidelberg, Germany, 103-114, doi: 10.1007/978-3-642-03211-0_9.

[18] Malandraki, C., Daskin, M.S. (1992). Time dependent vehicle routing problems: Formulations, properties and heuristic algorithms, Transportation Science, Vol. 26, No. 3, 185-200, doi: 10.1287/trsc.26.3.185.

[19] Hill, A.V., Benton, W.C. (1992). Modelling intra-city time-dependent travel speeds for vehicle scheduling problems, Journal of the Operational Research Society, Vol. 43, No. 4, 343-351, doi: 10.1038/sj/jors/0430406.

[20] Malandraki, C., Dial, R.B. (1996). A restricted dynamic programming heuristic algorithm for the time dependent traveling salesman problem, European Journal of Operational Research, Vol. 90, No. 1, 45-55, doi: 10.1016/03772217(94)00299-1.

[21] Ichoua, S., Gendreau, M., Potvin, J.-Y. (2003). Vehicle dispatching with time-dependent travel times, European Journal of Operational Research, Vol. 144, No. 2, 379-396, doi: 10.1016/s0377-2217(02)00147-9.

[22] Fleischmann, B., Gietz, M., Gnutzmann, S. (2004). Time-varying travel times in vehicle routing, Transportation Science, Vol. 38, No. 2, 160-173, doi: 10.1287/trsc.1030.0062.

[23] Huang, Y., Zhao, L., Van Woensel, T., Gross, J.-P. (2017). Time-dependent vehicle routing problem with path flexibility, Transportation Research Part B: Methodological, Vol. 95, 169-195, doi: 10.1016/i.trb.2016.10.013.

[24] Çimen, M., Soysal, M. (2017). Time-dependent green vehicle routing problem with stochastic vehicle speeds: An approximate dynamic programming algorithm, Transportation Research Part D: Transport and Environment, Vol. 54, 82-98, doi: 10.1016/i.trd.2017.04.016.

[25] Reil, S., Bortfeldt, A., Mönch, L. (2018). Heuristics for vehicle routing problems with backhauls, time windows, and 3D loading constraints, European Journal of Operational Research, Vol. 266, No. 3, 877-894, doi: 10.1016/ j.ejor.2017.10.029.

[26] Deb, K., Pratap, A., Agarwal, S., Meyarivan, T. (2002). A fast and elitist multiobjective genetic algorithm: NSGA-II, IEEE Transactions on Evolutionary Computation, Vol. 6, No. 2, 182-197, doi: 10.1109/4235.996017. 


\section{APEM}

Advances in Production Engineering \& Management

Letnik 14 | Številka 2 | Junij 2019 | Strani 201-212

https://doi.org/10.14743/apem2019.2.322
ISSN 1854-6250

Spletna stran: apem-journal.org Izvirni znanstveni članek

\title{
Časovno odvisen in dvokriterijski problem usmerjanja vozil s časovnimi okni
}

\author{
Zhao, P.X. ${ }^{a,}{ }^{,}$, Luo, W.H. ${ }^{a}$, Han, X. ${ }^{a}$ \\ ${ }^{a}$ School of Management, Shandong University, Jinan, Shandong, P.R. China
}

\section{POVZETEK}

Dvokriterijska optimizacija usmerjanja vozil je v zadnjih letih postala vroča raziskovalna tematika. $V$ tem prispevku je predlagan časovno odvisen in dvokriterijski problem usmerjanja vozil s časovnimi okni (TD-BO-VRPTW), kar je nova razširitev klasičnega problema usmerjanja vozil. Predstavljena je časovna odvisnost za situacije, pri katerih na hitrost vožnje vozila vplivata čas odhoda in razdalja med dvema strankama. Skupni stroški prevoza in časovni stroški sta dva kriterija, ki sta istočasno optimizirana z izgradnjo dvokriterijskega modela mešanega celoštevilskega linearnega programiranja. Za reševanje tega problema je uporabljen genetski algoritem z nedominiranim razvrščanjem II (NSGA-II), s katerim dobimo nabor Pareto optimalnih rešitev. V številčnih primerih je uporabljen primer RC108 iz Solomonovih standardnih testnih primerov, rezultati Pareto fronte pa kažejo na učinkovitost NSGA-II za TD-BO-VRPTW. Za preverjanje uspešnosti NSGA-II algoritma se oba kriterija optimizirata ločeno, nato pa je optimizirana še vsota obeh kriterijev. Če primerjamo te rezultate $\mathrm{z}$ rešitvami na Pareto fronti, je mogoče sklepati, da je algoritem zanesljiv, rezultati na Pareto fronti pa so konkurenčni, saj je prišlo do kompromisne rešitve med dvema ciljema.

\section{PODATKI O ČLANKU}

Ključne besede:

Težava usmerjanja vozil;

Časovna odvisnost;

Dvokriterijska optimizacija;

Časovna okna;

Optimalne rešitve Pareto;

Evolucijski algoritmi;

Algoritem NSGA-II

*Kontaktna oseba:

pxzhao@sdu.edu.cn

(Zhao, P.X.)

Zgodovina članka:

Prejet 14. februarja 2018

Popravljen 10. maja 2019

Sprejet 17. maja 2019 